\title{
Intercostal artery hemorrhage with hemothorax following combined lateral and posterior lumbar interbody fusion: a case report
}

\author{
Colleen Rentenberger ${ }^{1} \cdot$ Jennifer Shue ${ }^{1} \cdot$ Ellen M. Soffin ${ }^{1} \cdot$ Brendon M. Stiles $^{2} \cdot$ Chad M. Craig $^{1}$ • \\ Alexander P. Hughes ${ }^{1}$
}

Received: 4 April 2019 / Revised: 22 May 2019 / Accepted: 27 May 2019

(c) The Author(s), under exclusive licence to International Spinal Cord Society 2019

\begin{abstract}
Introduction Lateral lumbar interbody fusion (LLIF) is a safe treatment for degenerative spine conditions. However, risk of complications such as vascular injuries remains. We report a unique case of an intercostal artery (ICA) hemorrhage with hemothorax following LLIF.

Case presentation One hour after a right-sided LLIF L3-4 with posterior decompression L2-4 and L3-5 instrumentation, the patient became hypotensive, anemic and required vasopressor support. Evaluation revealed a right-sided hemothorax, which was caused by a bleeding intercostal artery, laterally at the 10th intercostal space. A lateral thoracotomy was performed to stop the bleeding. After vessel ligation and placement of two chest tubes, the patients' hemodynamics improved. The patient remained intubated overnight and was extubated on the first postoperative day.

Discussion Vascular injury is a rare complication of LLIF procedures. Most vascular injuries are segmental vessel lacerations, which resolve postoperatively. This is the first case description of ICA bleeding associated with LLIF surgery. Spontaneous ICA bleeding exists, but surgeons should be aware of careful handling in patients with vascular risk factors, especially with regard to patient positioning required in certain spinal surgical approaches. Timely vascular injury identification is critical for hemostasis and clinical management.
\end{abstract}

\section{Introduction}

Lateral interbody fusion is an overall safe treatment for various degenerative conditions. This procedure has also been used for thoracolumbar fractures with spinal cord or conus medullaris injuries [1,2]. Nonetheless, infection, visceral and neurologic injury may occur as perioperative complications [3]. Vascular complications are rare, but can be devastating especially when they occur in patients with spinal cord injuries due to altered physiological responses to hypovolemia. The reported lateral lumbar interbody fusion (LLIF) risk of a vascular injury ranges from 0.03 to $0.10 \%$

$\triangle$ Alexander P. Hughes

HughesA@hss.edu

1 Spine Care Institute, Hospital for Special Surgery, Weill Cornell Medical College, New York, NY, USA

2 Weill Cornell Brain and Spine Center, Division of Thoracic Surgery, New York-Presbyterian Hospital, Weill Cornell Medical College, New York, NY, USA
[3-5]. Most of the reported LLIF vascular complications are segmental vessel lacerations, which typically completely resolve postoperatively. We report a unique case of an intercostal artery (ICA) hemorrhage with hemothorax following LLIF.

\section{Case presentation}

A 56-year-old woman, BMI $21.5 \mathrm{~kg} / \mathrm{m}^{2}$, presented with constant lower back pain with bilateral lower extremity claudication from a motor vehicle accident 5 years prior. The patient reported a past surgical history of multiple spine surgeries with complex reconstruction at L4-5 in the 4 years prior without any relief. Other surgical history from 5 to 30 years prior included multiple left knee surgeries, pacemaker implantation, cholecystectomy and thyroid resection secondary to Graves' disease. Her past medical history included chronic obstructive pulmonary disease with chronic tobacco smoking (4 pack-year history), anxiety, depression, seizure disorder, and stage 3 chronic kidney disease. Review of systems revealed that she bruised easily, but had no family 
history of hemophiliac disease. Elevated blood pressure $(\sim 160 / 90 \mathrm{mmHg})$ was also noted. Her regular medications were oxcarbazepine, levothyroxine, a multivitamin and pain medication included prn use of acetaminophen with hydrocodone.

Radiographic examination depicted solid fusion through the L4-5 disc space, a degenerative retrolisthesis, moderate stenosis at L3-4, and a vacuum disc at L1-2 with no major coronal deformity. A right-sided LLIF L3-4 with posterior decompression L2-4 and L3-5 instrumentation was planned.

\section{Intraoperative course}

On the day of surgery, the patient was taken to the operating room and general anesthesia was induced with propofol $(2 \mathrm{mg} / \mathrm{kg})$ and fentanyl $(100 \mathrm{mcg})$. Vecuronium $(0.1 \mathrm{mg} / \mathrm{kg})$ was administered to facilitate direct laryngoscopy and intubation. A 7.0 endotracheal tube was placed without difficulty and secured. A right radial $20 \mathrm{Ga}$ arterial catheter and two additional peripheral intravenous catheters $(18 \mathrm{Ga}$ each) were placed. A right-sided transverse abdominis plane (TAP) block was placed under ultrasound guidance, using a $22 \mathrm{Ga} 2-3 / 8$ " Chiba needle ( $20 \mathrm{~mL} 0.5 \%$ bupivacaine with $2 \mathrm{mg}$ preservative free dexamethasone). The patient was positioned in a left lateral decubitus, right-sided elevated position. After blunt dissection through the abdominal muscles into the retroperitoneal space, the existing fusion at L4-5 was found to be intact. The LLIF was performed using standard technique at L3-4 [6]. After wound closure, the patient was converted to a prone position for the posterior stage of the operation.

Following dissection and division of the thoracolumbar fascia, an exploration of the L3-5 fusion was performed, which appeared unstable and therefore the existing hardware was removed. Revision screws were placed bilaterally at L3-5. A laminectomy with complete segmental decompression of L2-4 and decortication from L3-5 posterolateral elements was carried out. Hybrid graft and rods were placed for posterior fusion of L3-5. Subfascial drains were then applied and a standard layered closure was carried out.

On emergence from anesthesia, vital signs were stable, the patient moved all four extremities to command, and minute ventilation and pulmonary mechanics were appropriate for extubation. She was transferred to the post anesthesia care unit (PACU) for ongoing care. Over the subsequent hour in the PACU, the patient became progressively tachycardic, hypotensive, and poorly responsive. Bedside laboratory tests were significant for anemia and metabolic acidosis. A right-sided internal jugular vein central venous catheter was placed under ultrasound guidance and two units of autologous red blood cells (RBCs) and $500 \mathrm{~mL}$ of albumin were given. There was no improvement in hemodynamics, and epinepherine,

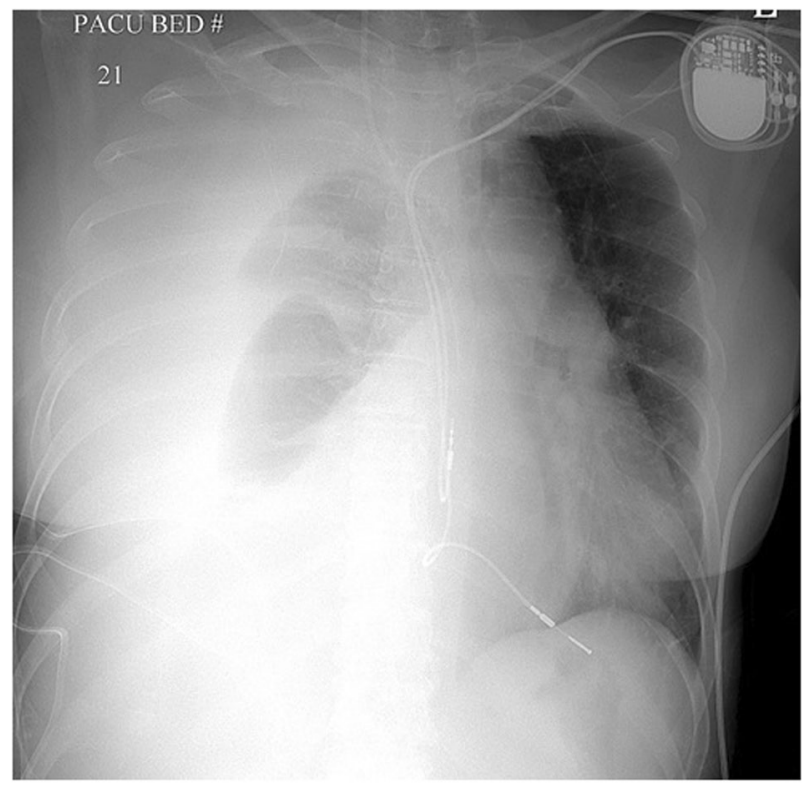

Fig. 1 Anteroposterior chest X-ray, performed in the recovery room after spine surgery demonstrating large right pleural effusion with shift of the mediastinum to the left side

vasopressin and phenylephrine infusions were required. Repeat laboratory testing revealed further drop in hemoglobin/hematocrit and elevated lactate. A presumptive diagnosis of hemorrhagic shock was made. The patient was emergently reintubated. Point-of-care ultrasound and transthoracic echocardiography examinations showed a massive right-sided hemothorax with evidence of impaired cardiac filling, and no retroperitoneal or abdominal bleeding. A portable chest X-ray confirmed right-sided effusion with leftward mediastinal shift (Fig. 1).

Cardiothoracic surgery was consulted. Prior to placing a chest tube, we activated the massive transfusion protocol and secured additional central venous access. The initial drainage from the chest tube was in excess of $2000 \mathrm{~mL}$ blood; the patient required 14 units of autologous RBCs, four units of fresh frozen plasma (FFP), and ongoing vasopressor support. The decision was made to return emergently to the operating room for thoracotomy and exploration.

A thoracic surgeon performed a lateral thoracotomy. The right hemithorax revealed an actively bleeding intercostal artery, laterally at the 10th intercostal space, which was ligated without complication. There was no evidence of rib fracture. After irrigating the right hemithorax, new chest tubes were placed and a layered closure was performed. Following the repair and prior to leaving the operating room, the patient was easily weaned from all vasopressor support. She was transported to the intensive care unit, intubated and sedated, with stable vital signs and hemoglobin/hematocrit. 


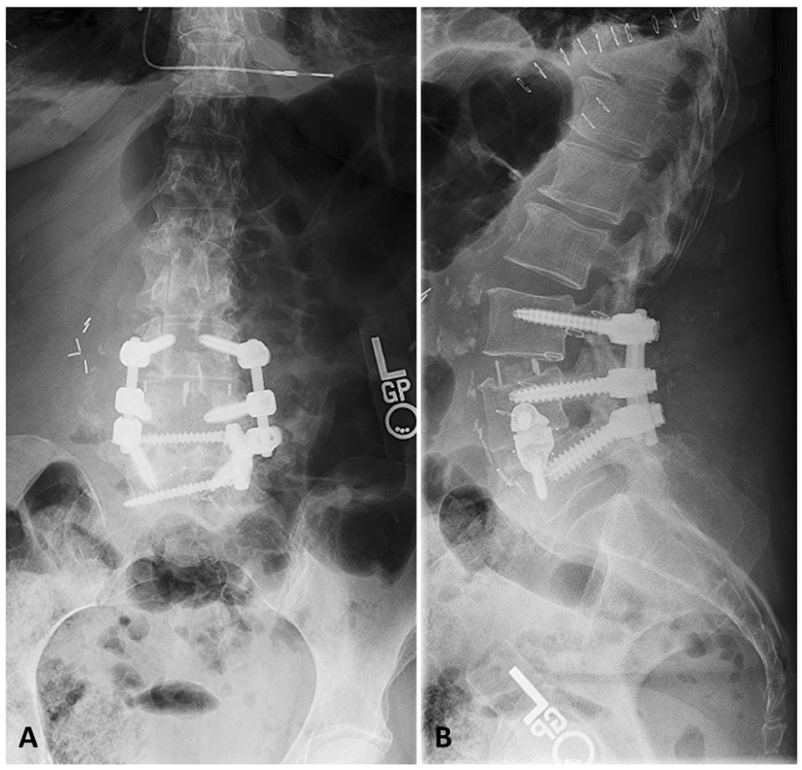

Fig. 2 Anteroposterior (a) and lateral (b) lumbar X-rays, performed 1 week after spine surgery illustrating satisfactory hardware positioning of interval interbody at L3-4 and instrumentation at L3-5

\section{Postoperative outcome}

The patient remained intubated overnight and was extubated on the first postoperative day (POD). Central venous access catheter drains and chest tubes were removed within 2 days after surgery. Consecutive chest X-rays showed a small right apical pneumothorax, which subsequently resolved prior to discharge. Her postoperative lab values showed no exceptionalities. To exclude any aneurysmal diseases, a chest computerized tomographic angiography on POD 9 was performed, which was negative for any aneurysmal deformations. Blood loss from the index surgery, during resuscitation, and the patient's postoperative course following emergent surgery required a total of 19 units of packed RBCs, $10 \mathrm{FFP}$, and five units of platelets. Overall the patient recovered slowly, but very well. The patient was discharged on POD 15 (Fig. 2).

At her 6 week postoperative follow-up the patient made significant improvement with no motor weaknesses, an unlimited ambulatory functional capacity and only a slight decreased sensation to her left foot. The radiographs showed solid maintenance of reconstruction. She continued to make excellent progress through her 3 month postoperative follow-up, with neither motor weakness nor sensory dysfunction and had unlimited walking capacity. The radiographs showed solid fusion of the operative segments.

\section{Discussion}

The risk of a vascular injury during LLIF is extremely rare. Kueper et al. reported an overall incidence of $0.056 \%$ per case and $0.029 \%$ per level at our institution [4]. Rodgers et al. has even reported no vascular injuries from his 600 cases [7]. Anatomically, segmental arteries and retroperitoneal vessels are the most likely to be injured during lumbar spine surgery $[4,8-12]$. The exact cause of the ICA bleeding in our case was not established. Vascular injuries can occur when retractors are positioned too anteriorly and if instrumentation migrates anterior to the disc space [5]. However, to our knowledge, ICA bleeding has not been reported in this context. Since the surgical procedure was not above the diaphragm and the retroperitoneal space showed no hematoma, it is also unlikely that the intercostal artery was directly injured during the spinal surgery. Another possible cause to consider is vessel injury during drain placement. However, the site of the hemorrhage was distant from where the drain was placed so it is unlikely that the drain trocar could have inadvertently transected the ICA in question.

Additionally, it is unlikely that the bleeding was caused by placement of central line or tube thoracostomy as the hemothorax occurred prior to these procedures. The preoperative peripheral nerve block is also an unlikely cause of bleeding. Besides being a safe procedure [13] due to ultrasound guidance technique, this kind of complication has never been described in the context of TAP. Furthermore, the needle used during this procedure would have been too short to reach an ICA from the abdomen.

There are only a few reported cases of spontaneous ICA bleeding in the literature [14-22]. To our knowledge, this is the first case description of ICA bleeding associated with LLIF surgery. Besides trauma, which is the most common reason for intercostal bleeding, predisposing factors include Neurofibromatosis Type 1, systemic lupus erythematosus (SLE), uncontrolled hypertension, anticoagulation and bleeding disorders $[15,16,19,20,22,23]$. Neurofibromatosis Type 1 is associated with arterial stenosis due to intimal or medial dysplasia and also arteriovenous malformations and aneurysms, which may lead to spontaneous vessel rupture in rare cases. SLE, being a chronic inflammatory disease with vasculitis as one of the main symptoms, can debilitate the vessels and make them more vulnerable to spontaneous rupture. Uncontrolled hypertension is also damaging to blood vessels, which become arteriosclerotic and therefore more rigid.

The most common location for spontaneous bleeding is the 10th and $11^{\text {th }}$ intercostal vessels [15], this might be because of the anatomical particularity of the 11th and 12th ribs being floating ribs and therefore more flexible [24]. The ICA bleeding at the 10th intercostal space that occurred subsequent to the LLIF spine surgery may have been coincidental. However, based on preoperative history and physical exam, the patient noted that she only bruised easily and did not have any physical exam evidence of ecchymosis 
or bleeding diathesis on exam objectively. The patient had untreated hypertension preoperatively. Additionally, she had a history of tobacco smoking and there was postoperative imaging evidence of obstructive lung disease, although the degree to which these pulmonary issues are related to her ICA bleeding are unclear. Her remaining medical history of bradycardia necessitating a left-sided permanent pacemaker in the past, hypothyroidism, and seizure disorder, are not known to be associated with ICA hemorrhage to our knowledge. She was also not on any medications associated with increased risk of perioperative bleeding surrounding spinal surgery, such as non-steroidal anti-inflammatory medications (including aspirin), anticoagulant or other antiplatelet agents (including serotonergic agents), vitamins or supplements, aside from a once daily multivitamin that she continued through surgery. One month prior to surgery she had one epidural steroidal injection (ESI) at L3-4. ESIs can lead to transient hypertension [25]. However, blood vessel fragility is more commonly associated with chronic steroid use and since the treated level was distal to the laceration, it is unlikely that the ESI played a major role in the bleeding.

As previously mentioned, arteriosclerosis is a consequence of hypertension. Nicotine use can enhance arteriosclerosis and due to its vasoconstrictive effects can lead to hypertension. The patient's smoking history and hypertension may have contributed to vessel fragility. Taking into account the patient's history, the most plausible cause of the bleeding was the patients' positioning and taping during the LLIF surgical procedure. To get a correct and stable lateral decubitus position, the patient must be fixated with tape to the table. Furthermore, for better access to the spine, the table has to be flexed so that the distance between the rib cage and the iliac crest increases [26]. During positioning of the patient, shear forces may have been generated that could have resulted in laceration of a fragile vessel. The delayed onset of the symptoms may be explained by the applied positive pressure due to artificial ventilation during the surgery. The positive pressure could have functioned similar to a tamponade such that the bleeding was symptomatic only after the extubation.

In conclusion, patient positioning is important in LLIF procedures, especially in patients with vascular risk factors. Surgeons should be particularly mindful with the taping and be cautious with breaking the table especially in predisposed patients. The availability of a thoracic or vascular surgeon for such cases may be lifesaving because timing is a key factor in identifying and stopping the bleed. Additionally, in spinal cord injury patients, special consideration should be made as they may be at higher risk for complications. Finally, this case highlights the importance of local resources and expertise including bedside evaluation with point-of-care ultrasound, echocardiography and blood bank services for favorable outcomes after unanticipated complications arising from LLIF.

Acknowledgements We would like to thank Ichiro Okano and Erika Chiapparelli for assistance with preparation of this manuscript.

\section{Compliance with ethical standards}

Conflict of interest CMC reports grants from Mallinckrodt Pharmaceuticals, and personal fees from Heron Therapeutics, with both relationships outside of the submitted work. APH reports personal fees from Altus Spine, other from Pfizer, Inc, grants from NuVasive, outside the submitted work. The remaining authors declare that they have no conflict of interests.

Publisher's note: Springer Nature remains neutral with regard to jurisdictional claims in published maps and institutional affiliations.

\section{References}

1. Malham GM. Minimally invasive direct lateral corpectomy for the treatment of a thoracolumbar fracture. J Neurol Surg Part A, Cent Eur Neurosurg. 2015;76:240-3.

2. Harrop JS, Hunt GE Jr., Vaccaro AR. Conus medullaris and cauda equina syndrome as a result of traumatic injuries: management principles. Neurosurg focus. 2004;16:e4.

3. Fujibayashi S, Kawakami N, Asazuma T, Ito M, Mizutani J, Nagashima $\mathrm{H}$, et al. Complications associated with lateral interbody fusion: Nationwide Survey of 2998 Cases during the first 2 years of its use in Japan. Spine (Philos Pa 1976). 2017;42:1478-84.

4. Kueper J, Fantini GA, Walker BR, Aichmair A, Hughes AP. Incidence of vascular complications during lateral lumbar interbody fusion: an examination of the mini-open access technique. Eur Spine J. 2015;24:800-9.

5. Uribe JS, Deukmedjian AR. Visceral, vascular, and wound complications following over 13,000 lateral interbody fusions: a survey study and literature review. Eur Spine J. 2015;24:386-96.

6. Salzmann SN, Shue J, Hughes AP. Lateral lumbar interbody fusion-outcomes and complications. Curr Rev Musculoskelet Med. 2017;10:539-46.

7. Rodgers WB, Gerber EJ, Patterson J. Intraoperative and early postoperative complications in extreme lateral interbody fusion: an analysis of 600 cases. Spine (Philos Pa 1976). 2011;36:26-32.

8. Aichmair A, Fantini GA, Garvin S, Beckman J, Girardi FP. Aortic perforation during lateral lumbar interbody fusion. J Spinal Disord Tech. 2015;28:71-5.

9. Assina R, Majmundar NJ, Herschman Y, Heary RF. First report of major vascular injury due to lateral transpsoas approach leading to fatality. J Neurosurg Spine. 2014;21:794-8.

10. Beckman JM, Vincent B, Park MS, Billys JB, Isaacs RE, Pimenta L, et al. Contralateral psoas hematoma after minimally invasive, lateral retroperitoneal transpsoas lumbar interbody fusion: a multicenter review of 3950 lumbar levels. J Neurosurg Spine. 2017;26:50-4.

11. Ganesan C, Petrus L, Ross IB. Regarding the possibility of anterior vascular injury from the posterior approach to the lumbar disc space: an anatomical study. Spine (Philos Pa 1976). 2012;37:1371-5.

12. Sakai T, Tezuka F, Wada K, Abe M, Yamashita K, Takata Y, et al. Risk management for avoidance of major vascular injury due 
to lateral transpsoas approach. Spine (Philos Pa 1976). 2016;41:450-3.

13. Abdallah FW, Chan VW, Brull R. Transversus abdominis plane block: a systematic review. Reg Anesth Pain Med. 2012;37:193-209.

14. Carr JA, Vadlamudi V, Azher QS. Intercostal artery aneurysmosis. Ann Thorac Surg. 2013;96:1870-3.

15. Dua A, Dua A, Jechow S, Desai SS, Kuy S. Idiopathic spontaneous rupture of an intercostal artery. Wmj. 2014;113:116-9.

16. Jang JY, Lim YS, Woo JH, Jang JH. Spontaneous rupture of intercostal artery after severe cough. Am J Emerg Med. 2015;33:131.e1-131.e3.

17. Lindbeck G. Abdominal wall hematoma in an elderly man. J Am Med Assoc. 1995;2/NO2:134-8.

18. Mathew R, Abdullah S, Renfrew I. Massive abdominal wall haematoma and haemothorax due to spontaneous rupture of an intercostal artery. Emerg Med J. 2008;25:607-8.

19. Moon JM, Lee SC, Chun BJ. Spontaneous intercostal artery bleeding. Emerg Med J. 2008;25:53-4.

20. Salmi R, Gaudenzi P, Ricci F, Gnani M, Morandi P, Di Todaro F. Sudden acute thoracic pain and hypovolemic shock as a presentation of spontaneous rupture of intercostal artery: a case report. J Am Geriatr Soc. 2012;60:371-3.

21. Sundram-Novelend SY, Appleton DS, Screaton NJ, Davies RJ, Chilvers ER. Spontaneous rupture of an intercostal artery aneurysm during air flight. Thorax. 2008;63:294.

22. Yu W, Wang C, Ng K, Chen W. Successful embolization of a ruptured intercostal artery after violent coughing. Am J Emerg Med. 2006;24:247-9.

23. Lu CC, Chen CH, Yeh SF, Lai JH, Chang DM. A spontaneous intercostal artery hemorrhage in systemic lupus erythematosus. Rheuma Int. 2012;32:829-31.

24. Lee LJ, Chang AT, Coppieters MW, Hodges PW. Changes in sitting posture induce multiplanar changes in chest wall shape and motion with breathing. Respir Physiol Neurobiol. 2010;170:236-45.

25. Younes M, Neffati F, Touzi M, Hassen-Zrour S, Fendri Y, Béjia I, et al. Systemic effects of epidural and intra-articular glucocorticoid injections in diabetic and non-diabetic patients. Jt Bone Spine. 2007;74:472-6.

26. Ozgur BM, Aryan HE, Pimenta L, Taylor WR. Extreme lateral interbody fusion (XLIF): a novel surgical technique for anterior lumbar interbody fusion. Spine J. 2006;6:435-43. 